\title{
OPTIMIZATION IN THE REGULARIZATION OF ILL-POSED PROBLEMS
}

\author{
A. R. DAVIES ${ }^{1}$ AND R. S. ANDERSSEN ${ }^{2}$
}

(Received 22 August 1985; revised 15 November 1985)

\begin{abstract}
We survey the role played by optimization in the choice of parameters for Tikhonov regularization of first-kind integral equations. Asymptotic analyses are presented for a selection of practical optimizing methods applied to a model deconvolution problem. These methods inciude the discrepancy principle, cross-validation and maximum likelihood. The relationship between optimality and regularity is emphasized. New bounds on the constants appearing in asymptotic estimates are presented.
\end{abstract}

\section{Introduction}

Optimization plays a crucial role in the regularization of ill-posed problems, in a variety of ways. For example, it does so in the choice of parameters appearing in the Tikhonov regularization of first-kind integral equations, in the computation of constrained regularized solutions to such equations when the solutions are known a priori to be in some closed convex set such as the sit of non-negative functions or the set of monotonic functions, and in a large number of image reconstruction problems where a function is to be estimated given only partial information about its Fourier transform or some similar set of observable linear functionals of it.

In this paper we examine one of these areas; we study the role of optimization in the Tikhonov regularization of first-kind integral equations. We develop our analysis for a model problem, that of inverting a Fredholm convolution equation

\footnotetext{
${ }^{1}$ Department of Applied Mathematics, University College of Wales, Aberystwyth SY23 3BZ, United Kingdom.

${ }^{2}$ Division of Mathematics and Statistics, C.S.I.R.O., P. O. Box 1965, Canberra City, A.C.T. 2601, Australia.

(C) Copyright Australian Mathematical Society 1986, Serial-fee code 0334-2700/86
} 
with periodic kernel. It has been shown by Anderssen and Prenter [2] that such a model serves as a useful framework for comparing different regularization techniques; in the present study we use our model to compare the performance of a selection of practical methods for estimating optimal parameters in Tikhonov regularization. Most of our results carry over to the general case of first-kind operator equations, and it is within this general setting that we introduce our discussion.

\subsection{Tikhonov regularization}

Consider an operator equation of the form

$$
K f=g \text {, }
$$

where $K$ is a mapping from a topological space $X$ to a topological space $Y$. We assume that $K$ is invertible but that the inverse mapping $K^{-1}$ is not continuous. Thus, for a given data function $g \in Y$, there exists a unique solution $f \in X$, but small perturbations (measured in the $Y$-topology) of the data can yield unbounded perturbations (measured in the $X$-topology) of the solution. The problem of inverting (1.1) is therefore ill-posed.

In the broadest sense, to regularize such a problem is simply to define a modified problem for which the inverse map is continuous and the solution of the modified problem (called the regularized solution) is an approximation to the solution of the original problem. A particular regularization method usually defines a family or sequence of modified problems parametrized in terms of one or more control variables, $\alpha$ say. In practice, a major consideration is how to select an optimal parameter set $\boldsymbol{\alpha}_{0}$, which in turn defines an optimal regularized solution $f_{\boldsymbol{\alpha}_{0}}$, say, among the family of possible regularized solutions $\left\{f_{\boldsymbol{\alpha}}\right\}$. Existing methods for selecting $\alpha$ on the whole attempt to optimize some estimated measure of the signal error $K f_{a}-g$.

In Tikhonov regularization (a detailed description may be found in Groetsch [10] and Tikhonov and Arsenin [16]) (1.1) is replaced by a well-posed problem of the form

$$
K_{\mathrm{a}} f_{\alpha}=g_{\alpha} \text {. }
$$

For simplicity, let us assume $K: L^{2} \rightarrow L^{2}$, where $L^{2}=L^{2}[0,1]$; also let $K$ be linear and compact. The regularized solution $f_{\alpha}$ is defined variationally as the minimizer of a functional of the form

$$
\Phi_{\alpha}(f)=\|K f-g\|^{2}+\lambda \Omega_{p}(f), \quad \alpha=(p, \lambda) .
$$

Here $\Omega_{p}$ is some non-negative stabilizing functional depending on a parameter $p \geqslant 0$ (called the order of regularization), and $\lambda>0$ is a constant (called the regularization parameter). A simple choice of $\Omega_{p}$ is

$$
\Omega_{p}(f)=\left\|f^{(p)}\right\|^{2} \text {, }
$$


where the norm in both (1.3) and (1.4) is $L^{2}$. In this case, the minimizer of (1.3) satisfies

$$
(\tilde{K} * \tilde{K}+\lambda I) f_{\alpha}=\tilde{K}^{*} g,
$$

where $\tilde{K}$ denotes the restriction of $K$ to the domain $H \subset L^{2}$, where $H$ is the completion of

$$
\left\{\phi: \phi^{(j)} \in L^{2}, j=0, \ldots, p-2 ; \phi^{(p-1)} \text { absolutely continuous }\right\}
$$

under the inner product

$$
\langle\phi, \psi\rangle=(-1)^{p}\left(\phi^{(2 p)}, \psi\right)_{L^{2}}+\sum_{j=1}^{p}(-1)^{p-J} \llbracket \phi^{(2 p-\jmath)} \psi^{(j)} \rrbracket,
$$

where $\llbracket \phi \rrbracket$ denotes $\phi(1)-\phi(0)$, and $\tilde{K}^{*}$ denotes the adjoint of $\tilde{K}$. Equation (1.5) is of the form (1.2) where $K_{\alpha}: H \rightarrow H$ is linear, invertible, and has a continuous inverse for $\lambda>0$.

The order of regularization, $p$, dictates the smoothness of the subspace $H$ in which the regularized solution $f_{\alpha}$ is sought, and also controls the sensitivity of $f_{\alpha}$ to perturbations in $g$. The regularization parameter $\lambda$, on the other hand, controls the trade-off in (1.3) between minimizing the residual and stabilizing the solution. If $\lambda$ is too small, the original ill-posed problem (1.1) will tend to dominate, and therefore numerical errors will be catastrophically amplified; if $\lambda$ is too large, the regularized solution will not adequately satisfy (1.1). We therefore seek to optimize $\boldsymbol{\alpha}=(p, \lambda)$.

\subsection{Optimizing $\alpha$}

In theory we might define an absolutely optimal $\alpha$ as that which minimizes the error

$$
\left\|f_{\alpha}-f\right\| \text {. }
$$

Let us first consider the choice of $p$. An absolutely optimal $p$ should depend on the smoothness of the true solution $f$ and the decay-rate of the spectrum of $K$. (For theoretical studies in support of this, see for example Cullum [5] and Lukas $[13,14]$.) In practice, however, the smoothness of $f$ is usually unknown, and the optimization of $p$ using a practical method which attempts to minimize an estimate of (1.6) is not possible. One is forced instead to attempt to minimize estimates of the signal error

$$
\left\|K f_{\mathbf{\alpha}}-g\right\|
$$

with respect to $p$ and $\lambda$. An interesting pragmatic approach was studied by Lukas [13]; others have used generalized cross-validation and maximum likelihood estimation in this context (Baart [3], Davies et al [8] and Gamber [9]). In each 
case a certain function of $\alpha$ is minimized with respect to $\lambda$ for a sequence of $p$-values and hence a minimizing pair $(p, \lambda)$ is found. Observations on the efficacy of such procedures are made in the sequel.

Consider now the choice of $\lambda$ for fixed $p$. When the data function is inexact, which is always the case in practical situations, the choice of $\lambda$ is often critical. In the numerical experiments of de Hoog [11] it is shown that the best choice of $\lambda$ can depend quite crucially on the actual errors present in the data rather than just their statistical properties. Any method of optimizing $\lambda$ should therefore take account of the data being analyzed.

Again, it is difficult in practice to find an absolutely optimal value of $\lambda$ (in the sense of minimizing (1.6) for fixed $p$ ), and we rely on optimizing estimates of (1.7). Practical methods fall into two classes, those which require a priori estimates of the variances of the noise in the data, and those which do not. In what follows we study two methods in the former class, based on the discrepancy principle $[10,16]$ much favoured by Soviet workers, and two methods in the latter class, namely cross-validation and maximum likelihood. In each case we examine the optimality properties of the estimated regularization parameters and compare them with the true minimizer of (1.7).

Most of the detailed asymptotic results presented in this paper appear here for the first time, although the associated rates of convergence are on the whole well-known. A note on proof techniques is given in an appendix, and illustrated by sketch proofs of Theorems 3.1 and 3.2.

\section{The model problem}

Consider the Fredholm convolution integral equation of the first kind given by

$$
(K f)(x) \equiv \int_{0}^{1} k(x-y) f(y) d y=g(x), \quad 0 \leqslant x \leqslant 1,
$$

where $g$ is measured discretely with random errors. For simplicity we assume

(a) $f, g$ and $k \in L^{2}$, and each is periodic with period 1;

(b) the Fourier coefficients of $k$, defined by

$$
\hat{k}_{q}=\int_{0}^{1} k(x) \exp \left(-i \omega_{q} x\right) d x, \quad \omega_{q}=2 \pi q,
$$

satisfy

$$
\hat{k}_{q} \neq 0, \quad q=0, \pm 1, \pm 2, \ldots .
$$

This means that $K$ is invertible but $K^{-1}$ is not continuous since $\hat{k}_{q} \rightarrow 0$ as $q \rightarrow \pm \infty$. 
(c) The sampled data are

$$
y_{n}=g\left(x_{n}\right)+\varepsilon_{n}, \quad n=0,1, \ldots, N-1,
$$

with uniform sample points $x_{n}=n / N$;

(d) the errors satisfy

$$
E \varepsilon_{n}=0, \quad E \varepsilon_{l} \varepsilon_{n}=\sigma^{2} \delta_{l n}, \quad l, n=0, \ldots, N-1,
$$

where $E$ denotes expectation with respect to the error distribution. This model problem can be treated very simply in terms of the discrete Fourier transform (DFT), while it incorporates all the intrinsic difficulties of more general operator equations of the first kind.

For convenience let $N$ be even and $M=\frac{1}{2} N$. The DFT of any discrete set $\left\{\phi_{n}\right\}_{n=0}^{N-1}$ is then defined by

$$
\hat{\phi}_{N, q}=\frac{1}{N} \sum_{n=0}^{N-1} \phi_{n} \exp \left(-i \omega_{q} x_{n}\right), \quad-M \leqslant q \leqslant M,
$$

and has the property

$$
\hat{\boldsymbol{\phi}}_{N,-q}=\overline{\hat{\boldsymbol{\phi}}_{N, q}}, \quad 0 \leqslant q \leqslant M,
$$

whenever the $\phi_{n}$ are real-valued. The coefficients in (2.5) give rise to the real-valued trigonometric interpolant

$$
\phi_{N}(x)=\sum_{q=-M}^{M} \hat{\phi}_{N, q} \exp \left(i \omega_{q} x\right), \quad 0 \leqslant x \leqslant 1,
$$

where the interpolation takes place at the sample points $x_{n}$, i.e.

$$
\phi_{N}\left(x_{n}\right)=\phi_{n}, \quad n=0, \ldots, N-1 \text {. }
$$

(The notation $\Sigma^{\prime \prime}$ denotes half-weighting of the first and last terms in the sum.) In the sequel, when $\phi=\phi(x)$ we denote $\phi\left(x_{n}\right)$ by $\phi_{n}$, so that $\phi_{N}$ given by (2.7) interpolates $\phi$. The vector $\phi_{N} \in \mathbf{R}^{N}$ denotes $\left(\phi_{0}, \ldots, \phi_{N-1}\right)^{T}$.

Let $T_{M}$ denote the space of real-valued trigonometric polynomials of degree at most $M$ and period 1 . We shall study regularized solutions in this space. We note the following elementary results:

Lemma 2.1 (i) If $\phi \in T_{M}$ then $K \phi \in T_{M}$ and

$$
(K \phi)(x)=\left(K \phi_{N}\right)(x)=\sum_{q=-M}^{M} \hat{k}_{q} \hat{\phi}_{N, q} \exp \left(i \omega_{q} x\right) .
$$

(ii) If $\phi \in L^{2}$ is the solution of

then $\phi \in T_{M}$ and

$$
K \phi=g_{N}
$$

$$
\phi(x)=\phi_{N}(x)=\sum_{q=-M}^{M} \hat{k}_{q}^{-1} \hat{g}_{N, q} \exp \left(i \omega_{q} x\right) .
$$


(iii) The solution $\phi$ of (2.10) interpolates $\phi_{N}$ which satisfies

$$
K_{N} \phi_{N}=\mathbf{g}_{N},
$$

where $K_{N}$ is the $N \times N$ circulant matrix

$$
\left.\begin{array}{l}
K_{N}=\frac{1}{N} \Psi \hat{K}_{N} \Psi^{H} \\
\hat{K}_{N}=\operatorname{diag}\left(\hat{k}_{0}, \hat{k}_{1}, \ldots, \hat{k}_{M}, \hat{k}_{-M+1}, \ldots, \hat{k}_{-1}\right) \\
\Psi=\left(\Psi_{r s}\right), \Psi_{r s}=\exp (2 \pi i r s / N), r, s=0, \ldots, N-1 .
\end{array}\right\}
$$

In the above setting, the unregularized model problem can be written: find $f_{N} \in T_{M}$ such that

$$
K_{N} \mathbf{f}_{N}=\mathbf{y}_{N}, \quad \text { where } \mathbf{y}_{N}=\left(y_{0}, y_{1}, \ldots, y_{N-1}\right) .
$$

Under $p$ th-order Tikhonov regularization we seek

$$
\min _{f \in T_{M}}\left\{\frac{1}{N} \sum_{n=0}^{N-1}\left[(K f)\left(x_{n}\right)-y_{n}\right]^{2}+\lambda\left\|f^{(p)}\right\|^{2}\right\},
$$

or equivalently

$$
\min _{\hat{f}_{N}} \sum_{q=-M}^{M}\left\{\left|\hat{k}_{q} \hat{f}_{N, q}-\hat{y}_{N, q}\right|^{2}+\lambda \omega_{q} 2 p\left|\hat{f}_{N, q}\right|^{2}\right\},
$$

where $\hat{\mathbf{f}}_{N} \in \mathbf{C}^{N}$ has elements

$$
\left(\hat{f}_{0}, \hat{f}_{N, 1}, \ldots, \hat{f}_{N, M}, \hat{f}_{N,-M+1}, \ldots, \hat{f}_{N,-1}\right)^{T}
$$

which satisfy (2.6), and $\hat{\mathbf{y}}_{N}$ is the DFT of $\mathbf{y}_{N}$. It is easily shown that the minimizer $f_{N ; \propto}$ of (2.15) may be written (cf. (1.5))

$$
f_{N ; \boldsymbol{\alpha}}(x)=\sum_{q=-M}^{M} \hat{f}_{N, q ; \boldsymbol{\alpha}} \exp \left(i \omega_{q} x\right), \quad 0 \leqslant x \leqslant 1,
$$

where

$$
\hat{f}_{N, q ; \alpha}=Z_{q ; \alpha} \hat{y}_{N, q} / \hat{k}_{q}, \quad-M \leqslant q \leqslant M,
$$

with

$$
Z_{q, a}=\left(1+\lambda \omega_{q} 2 p /\left|\hat{k}_{q}\right|^{2}\right)^{-1}, \quad-M \leqslant q \leqslant M .
$$

The numbers $Z_{q, \propto}$ in (2.18)-(2.19) constitute a discrete regularization filter depending on the parameter set $\alpha=(p, \lambda)$ which is to be optimized. The stabilizing effect results from the properties

(i) $0<Z_{q ; \alpha} \leqslant 1$, and (ii) $Z_{q ; \alpha} \rightarrow 0$ as $q \rightarrow \pm \infty, \lambda>0$.

Using Lemma 2.1 we can show that $f_{N ; a}$ interpolates the solution of the discrete problem (cf. (1.2))

$$
K_{N ; \boldsymbol{\alpha}} \mathbf{f}_{N ; \boldsymbol{\alpha}}=\mathbf{y}_{N}
$$


where

$$
K_{N ; \alpha}=\frac{1}{N} \Psi \hat{K}_{N} \hat{A}^{-1}(\alpha) \Psi^{H}
$$

and

$$
\hat{A}(\boldsymbol{\alpha})=\operatorname{diag}\left(1, Z_{1, \alpha}, \ldots, Z_{M, \alpha}, Z_{M-1, \alpha}, \ldots, Z_{1, \alpha}\right) .
$$

The predictive signal $g_{N ; \boldsymbol{\alpha}}=K f_{N ; \boldsymbol{\alpha}}$ can be seen to interpolate the vector

$$
\mathbf{g}_{N ; \boldsymbol{\alpha}}=A(\boldsymbol{\alpha}) \mathbf{y}_{N},
$$

where

$$
A(\alpha)=\frac{1}{N} \Psi \hat{A}(\alpha) \Psi^{H} .
$$

is known as the influence matrix of the regularized problem.

\section{Optimality and regularity}

An absolutely optimal $\alpha$ minimizes the predictive mean-square error

$$
\frac{1}{N} \sum_{n=0}^{N-1}\left[f_{N ; \mathbf{\alpha}}\left(x_{n}\right)-f\left(x_{n}\right)\right]^{2}
$$

but, as we have pointed out previously, it is difficult to obtain a practical estimate of this minimizer in the absence of detailed information concerning $f$. Consider instead the predictive mean-square signal error

$$
S(\boldsymbol{\alpha})=\frac{1}{N} \sum_{n=0}^{N-1}\left[g_{N ; \boldsymbol{\alpha}}\left(x_{n}\right)-g\left(x_{n}\right)\right]^{2} .
$$

The minimizer of $S(\alpha)$ is estimated quite closely by several practical statistical methods, at least when $p$, the order of regularization, is fixed. We shall study the asymptotic nature of these estimates as $N \rightarrow \infty$.

For the present, let $p$ be fixed. We emphasize this by writing $\lambda$ wherever previously we have used $\alpha$. Let $\lambda_{0}$ denote the minimizer of $S(\lambda)$. Following Davies and Anderssen [7] we say that a value of $\lambda$ is

(i) $S$-optimal if $S(\lambda) / S\left(\lambda_{0}\right)=1+o(1)$ as $N \rightarrow \infty$,

where $o(1) \rightarrow 0$ as $N \rightarrow \infty$;

(ii) weakly S-optimal if $S(\lambda) / S\left(\lambda_{0}\right)=O(1)$ as $N \rightarrow \infty$;

(iii) S-suboptimal if $S(\lambda) / S\left(\lambda_{0}\right) \rightarrow \infty$ as $N \rightarrow \infty$.

(In [7] a stricter form of (3.3a) is also introduced. This strong optimality will not be discussed in the present paper.) 
The definitions (3.3) can, of course, be extended to loss functions other than (3.2), for example (3.1). It is important to realize that a $\lambda$ which is $S$-optimal will not, in general, be absolutely optimal; indeed, an $S$-optimal $\lambda$ is normally absolutely suboptimal (see [7]). Nevertheless, both $S$-optimal and weakly $S$-optimal estimates of $\lambda$ have been used extensively in practice with some success, at least when the problems under study are not too severely ill-posed (see, for example, [8]).

Let $\tilde{\lambda}_{0}$ denote the minimizer of $E S(\lambda)$. We maintain the definitions (3.3) if $S(\lambda)$ is replaced by $E S(\lambda)$ and $\lambda_{0}$ by $\tilde{\lambda}_{0}$. In the problem of spline smoothing $(K=I$ in (1.3)), a number of authors have studied the asymptotic properties of $\bar{\lambda}_{0}$ and $E S\left(\tilde{\lambda}_{0}\right)$ (see Wahba [21]) under a variety of regularity conditions on the data. In the present problem we must also take account of the spectral decay of the kernel.

Following Wahba [20] and Lukas [14] we will assume an algebraic decay:

Aхіом A: There exists a number $m \geqslant 1$ such that

$$
\left|\hat{k}_{q}\right|=c_{q}^{-1} \omega_{q}^{-m}, \quad 0<c \leqslant c_{q} \leqslant C<\infty, \quad q=1,2, \ldots,
$$

where $c$ and $C$ are constants.

Moreover, let $\nu=m+p$, and consider the Fourier coefficients $\hat{g}_{q}$ (cf. (2.2)) of the underlying data function. We will consider two alternative regularity conditions on the data:

Aхіом B (strong regularity).

$$
\sum_{q=0}^{\infty} \omega_{q}^{4 \nu}\left|\hat{g}_{q}\right|^{2}<\infty
$$

Aхıом C (weak regularity):

$$
\sum_{q=0}^{\infty} \omega_{q}^{2 \nu}\left|\hat{g}_{q}\right|^{2}<\infty
$$

We also introduce the ratio

$$
\rho=C / c \geqslant 1
$$

and the measures

$$
\|g\|_{r}=\frac{1}{\pi} \int_{0}^{\infty} \omega^{2 r}|\hat{g}(\omega)|^{2} d \omega, \quad r>0
$$

whenever the integrals converge, where

$$
\hat{g}(\omega)=\int_{0}^{1} g(x) \exp (-i \omega x) d x .
$$


We can now state the following (cf. Theorem 3.1 in [7]):

THEOREM 3.1 Under Axioms A and $\mathrm{B}$, and $g_{N ; \lambda} \in T_{M}$, the value of $\lambda$ which minimizes $E S(\lambda)$ may be written

$$
\tilde{\lambda}_{0}=\theta_{0} \cdot\left[\frac{\kappa_{\nu}}{4 \nu} \cdot \frac{\sigma^{2}}{\|g\|_{2 \nu}} \cdot \frac{1}{N}\right]^{2 \nu /(4 \nu+1)}[1+o(1)] \text { as } N \rightarrow \infty,
$$

where

$$
\rho^{-4 \nu /(4 v+1)} C^{-2} \leqslant \theta_{0} \leqslant \rho^{4 \nu /(4 v+1)} c^{-2},
$$

$\kappa_{\nu}$ is the constant

$$
\kappa_{\nu}=\frac{1}{\pi} \int_{0}^{\infty} \frac{d t}{\left(1+t^{2 \nu}\right)^{2}},
$$

and $o(1) \rightarrow 0$ as $N \rightarrow \infty$. The minimizing value of $\operatorname{ES}(\lambda)$ as $N \rightarrow \infty$ is

$$
E S\left(\tilde{\lambda}_{0}\right)=\phi_{0} \cdot\left[\frac{\kappa_{\nu}}{4 \nu} \cdot \frac{\sigma^{2}}{N}\right]^{4 \nu /(4 \nu+1)}\|g\|_{2 \nu}^{1 /(4 \nu+1)}[1+o(1)]
$$

where

$$
\begin{aligned}
& 4 \nu R^{-1}+R^{-4 \nu} \leqslant \phi_{0} \leqslant 4 \nu R+R^{4 \nu}, \\
& R^{\nu}=\rho^{(6 \nu+1) /(4 \nu+1)} .
\end{aligned}
$$

Theorem 3.2 Under Axioms $\mathrm{A}$ and $\mathrm{C}$,

$$
\tilde{\lambda}_{0} \geqslant \theta_{0} \cdot\left[\frac{\kappa_{v}}{4 \nu} \cdot \frac{\sigma^{2}}{\|g\|_{\nu}} \cdot \frac{1}{N}\right]^{2 \nu /(2 \nu+1)}[1+o(1)] \text { as } N \rightarrow \infty
$$

where

$$
\rho^{-8 \nu /(2 \nu+1)} C^{-2} \leqslant \theta_{0} \leqslant \rho^{8 \nu /(2 \nu+1)} c^{-2},
$$

and

$$
\operatorname{ES}\left(\tilde{\lambda}_{0}\right) \leqslant \phi_{0} \cdot\left(\frac{\kappa_{\nu}}{2 \nu} \cdot \frac{\sigma^{2}}{N}\right)^{2 \nu /(2 v+1)}\|g\|_{\nu}^{1 /(2 v+1)}[1+o(1)]
$$

where

$$
\begin{aligned}
& 2 \nu R^{-1}+R^{-2 \nu} \leqslant \phi_{0} \leqslant 2 \nu R+R^{2 \nu}, \\
& R^{2 \nu}=\rho^{(8 \nu+1) /(2 \nu+1)} .
\end{aligned}
$$

The expressions (3.13) and (3.17) give some theoretical indication of how the minimizing value of $S(\lambda)$ depends on $p, \sigma^{2}, m$ (the smoothness of the kernel), and the regularity of $g$. In particular, we may make deductions concerning the existence of an optimal $p$. Under strong regularity, a brief examination of (3.13) 
shows that, as $p$ is increased, the value of $\operatorname{ES}\left(\tilde{\lambda}_{0}\right)$ is determined essentially by the term $\|g\|_{2 \nu}^{1 /(4 \nu+1)}$. This will become divergent for $p$ sufficiently large, unless $g$ is infinitely regular (i.e. $\|g\|_{r}<\infty$ for all $r>0$ ), and so an optimal value of $p$ clearly exists which minimizes $E S(\alpha)$. On the other hand if $g$ is infinitely regular then we can distinguish two possibilities. Either $\|g\|_{2 \nu}$ increases with $p$ at a rate greater than (constant) ${ }^{4 \nu+1}$, in which case $E S\left(\tilde{\lambda}_{0}\right)$ must eventually increase with $p$ and so an optimal $p$ must exist, or $\|g\|_{2 v}$ increases with $p$ at a rate not exceeding (constant) ${ }^{4 \nu+1}$, in which case the dependence of $E S\left(\tilde{\lambda}_{0}\right)$ on $p$ may be only marginal.

When $g$ is only weakly regular, then increasing $p$ must quickly lead to the divergence of $\|g\|_{\nu}$, and hence that of the upper bound in (3.17). Thus, if the rates in this bound are achieved, an optimal $p$ must exist.

\section{Practical optimizing methods for $\lambda$ when $\sigma^{2}$ is known}

In practice $S(\lambda)$ cannot be computed directly without knowing the exact data function, and so alternative means of estimating its minimizer are needed. Let $p$ be fixed, and consider the predictive mean-square error

$$
D\left(\lambda ; y_{N}\right)=\frac{1}{N} \sum_{n=0}^{N-1}\left[K f_{N ; \lambda}\left(x_{n}\right)-y_{n}\right]^{2}
$$

sometimes called the (squared) discrepancy in the approximate solution $f_{N ; \lambda}$. It is easy to show that, on average, $D\left(\lambda ; \mathbf{y}_{N}\right)$ is an overestimate of $S(\lambda)$ as $N \rightarrow \infty$. Moreover, (4.1) is directly computable in the form

$$
D\left(\lambda ; \mathbf{y}_{N}\right)=\frac{1}{N}\left\|(I-A(\lambda)) \mathbf{y}_{N}\right\|^{2}=\left\|(I-\hat{A}(\lambda)) \hat{\mathbf{y}}_{N}\right\|^{2} .
$$

A simple calculation shows that

$$
E D\left(\lambda ; \mathbf{y}_{N}\right)=\sum_{q=-M}^{M}\left(1-Z_{q ; \lambda}\right)^{2}\left|\hat{g}_{N, q}\right|^{2}+\frac{\sigma^{2}}{N} \sum_{q=-M}^{M}\left(1-Z_{q ; \lambda}\right)^{2},
$$

whereas, from (3.2),

$$
E S(\lambda)=\sum_{q=-M}^{M}\left(1-Z_{q ; \lambda}\right)^{2}\left|\hat{g}_{N, q}\right|^{2}+\frac{\sigma^{2}}{N} \sum_{q=-M}^{M} Z_{q ; \lambda}^{2} .
$$

It is then not difficult to deduce that under Axiom $\mathrm{A}$

$$
E D\left(\lambda ; y_{N}\right)=E S(\lambda)+\sigma^{2}-O\left(\left[N \lambda^{1 /(2 \nu)}\right]^{-1}\right)
$$

provided $N \lambda^{1 /(2 v)} \rightarrow \infty$ as $N \rightarrow \infty$. This is certainly true for any fixed $\lambda>0$. 


\subsection{The discrepancy principle}

When the variance $\sigma^{2}$ is known, Soviet mathematicians have widely favoured choosing $\lambda=\lambda\left(\sigma^{2}\right)$ so that the discrepancy should be in agreement with the errors in the data, i.e.,

$$
D\left(\lambda ; \mathbf{y}_{N}\right)=\sigma^{2} .
$$

(There is no point in minimizing $D$ with respect to $\lambda$ since the minimizer is $\lambda=0$, which corresponds to an unregularized problem.) The discrepancy principle embodied in (4.6) is an easily implemented a posteriori strategy for choosing $\lambda$. It was first clearly enunciated by Morozov [15], although it had been used earlier in an intuitive way by others. See $[10,16]$ for a detailed description.

We can see that (4.6) has a unique solution for given $\mathbf{y}_{N}$ since, from (4.2), $D(\lambda$; $\left.\mathbf{y}_{N}\right)$ is an increasing function of $\lambda$ with $D\left(0 ; \mathbf{y}_{N}\right)=0$ and $D\left(\lambda ; \mathbf{y}_{N}\right) \rightarrow \frac{1}{N}\left\|\mathbf{y}_{N}\right\|^{2}$ as $\lambda \rightarrow \infty$, this limit clearly exceeding $\sigma^{2}$ unless the data is totally dominated by noise. We now state

THEOREM 4.1 Under Axioms A and B the solution of the nonlinear equation

$$
E D\left(\lambda ; \mathbf{y}_{N}\right)=\sigma^{2}
$$

may be written

$$
\tilde{\lambda}_{D}=\theta_{D} \cdot\left[\frac{\sigma^{2}}{\|g\|_{2 v}} \cdot \frac{1}{N}\right]^{2 \nu /(4 v+1)}[1+o(1)] \quad \text { as } N \rightarrow \infty
$$

where $o(1) \rightarrow 0$ and

$$
\begin{gathered}
C^{-2}\left[\kappa_{\nu}+\left(1+\rho^{-2}\right) \mu_{\nu 2}\right]^{2 \nu /(4 \nu+1)} \leqslant \theta_{D} \leqslant c^{-2}\left[\kappa_{\nu}+\left(1+\rho^{2}\right) \mu_{\nu 2}\right]^{2 \nu /(4 \nu+1)}, \\
\mu_{\nu 2}=\frac{1}{\pi} \int_{0}^{\infty} \frac{t^{2 \nu} d t}{\left(1+t^{2 \nu}\right)^{2}}
\end{gathered}
$$

Under strong regularity, therefore, $\tilde{\lambda}_{D}$ has the same rate of convergence as $\tilde{\lambda}_{0}$. This means that $\lambda_{D}$ is weakly $S$-optimal. In particular we may deduce

$$
a_{\nu}\left(\rho^{-1}\right) \leqslant \tilde{\lambda}_{D} / \tilde{\lambda}_{0} \leqslant a_{\nu}(\rho),
$$

where

$$
a_{\nu}(\rho)=\rho^{(12 \nu+2) /(4 \nu+1)}\left[4 \nu\left\{1+\frac{\mu_{\nu 2}}{\kappa_{\nu}}\left(1+\rho^{2}\right)\right\}\right]^{2 \nu /(4 \nu+1)}[1+o(1)] .
$$

In the case $\rho=1$, which describes the problem of numerical differentiation, we have

$$
\tilde{\lambda}_{D}=\tilde{\lambda}_{0}\left[4 \nu\left(1+2 \mu_{\nu 2} / \kappa_{\nu}\right)\right]^{2 \nu /(4 \nu+1)}[1+o(1)]
$$


This means that $\tilde{\lambda}_{D}$ considerably oversmooths relative to $\tilde{\lambda}_{0}$, particularly as $p$ is increased. This result generalizes an earlier result in Wahba [19].

THEOREM 4.2 Under Axioms A and C, the solution of (4.7) satisfies

$$
\tilde{\lambda}_{D} \geqslant \theta_{D} \cdot\left[\frac{\sigma^{2}}{\|g\|_{\nu}} \cdot \frac{1}{N}\right]^{2 v /(2 v+1)}[1+o(1)] \text { as } N \rightarrow \infty,
$$

where

$$
C^{-2}\left[\kappa_{\nu}+\left(1+\rho^{-2}\right) \mu_{\nu 2}\right]^{2 \nu /(2 \nu+1)} \leqslant \theta_{D} \leqslant c^{-2}\left[\kappa_{\nu}+\left(1+\rho^{2}\right) \mu_{\nu 2}\right]^{2 \nu /(2 \nu+1)} .
$$

If the rates in (3.15) and (4.13) are achieved by $\bar{\lambda}_{0}$ and $\tilde{\lambda}_{D}$, respectively, then we may again deduce that $\lambda_{D}$ is weakly $S$-optimal under weak regularity. The actual ratio $\bar{\lambda}_{D} / \tilde{\lambda}_{0}$ is, however, more difficult to estimate in this case.

\subsection{A Bayesian approach}

A different way of exploiting the discrepancy principle which leads to slightly improved estimates of $\lambda_{0}$ is to replace $D$ in (4.6) by its expectation with respect to an a posteriori distribution. Such an approach was proposed by Turchin [17, 18]. Consider an a priori conditional distribution for $\mathbf{y}_{N}$ with density function

$$
P\left(\mathbf{y}_{N} \mid \mathbf{f}_{N}\right)=\prod_{n=0}^{N-1} \frac{1}{\sigma \sqrt{2 \pi}} \exp \left[-\left|\left(K_{N} \mathbf{f}_{N}\right)_{n}-y_{n}\right|^{2} /\left(2 \boldsymbol{\sigma}^{2}\right)\right]|J|
$$

where $J$ is the Jacobian of the transformation from $\left(K_{N} \mathbf{f}_{N}\right)_{n}$ to $f_{n}$, assumed independent of $n$. If we define an a priori distribution for $f_{N}$ by means of the parametrized density function

$$
P_{\alpha}\left(f_{N}\right)=\prod_{\substack{q=-M \\ q \neq 0}}^{M-1}\left(N \lambda \omega_{q}^{2 p} / \sigma^{2}\right) \exp \left[-N \lambda \omega_{q}^{2 p}\left|\hat{f}_{N, q}\right|^{2} / \sigma^{2}\right],
$$

then the a posteriori conditional distribution for $\mathbf{f}_{N}$ has the density function

$$
P\left(\mathbf{f}_{N} \mid \mathbf{y}_{N}\right)=\text { constant. } \prod_{\substack{q=-M \\ q \neq 0}}^{M-1} \exp \left[-\frac{N}{\boldsymbol{\sigma}^{2}}\left\{\left|\hat{k}_{q} \hat{f}_{N, q}-\hat{y}_{N, q}\right|^{2}+\lambda \omega_{q}^{2 p}\left|\hat{f}_{N, q}\right|^{2}\right\}\right] \text {. }
$$

If we denote expectation with respect to $P\left(f_{N} \mid y_{N}\right)$ by $\mathscr{E}$ it can be shown that (using techniques similar to those found in[17])

$$
\mathscr{E} \mathbf{f}_{N}=\Psi \hat{\mathbf{f}}_{N ; \boldsymbol{\alpha}}
$$

where the matrix $\Psi$ is defined in (2.13) and the elements of $\hat{\mathbf{f}}_{N ; \boldsymbol{\alpha}}$ are given by (2.18)-(2.19). The interpolant of $\mathscr{E} \mathbf{f}_{N}$ in $T_{M}$ is therefore the regularized solution (2.17). The expectation of the discrepancy is

$$
\tilde{D}\left(\boldsymbol{\alpha} ; \mathbf{y}_{N}\right)=\mathscr{E} \frac{1}{N}\left\|K_{N} \mathbf{f}_{N}-\mathbf{y}_{N}\right\|^{2}
$$


which, after some calculation, reduces to

$$
\tilde{D}\left(\boldsymbol{\alpha} ; \mathbf{y}_{N}\right)=\frac{1}{N}\left\{\left\|(I-A(\alpha)) \mathbf{y}_{N}\right\|^{2}+\left(\sigma^{2} / N\right) \operatorname{Trace} A(\alpha)\right\}>D\left(\alpha ; \mathbf{y}_{N}\right) .
$$

We now state the following interesting result:

THEOREM 4.3 Under Axioms A and $\mathrm{B}$, the solution of the nonlinear equation

$$
E \tilde{D}\left(\lambda ; \mathbf{y}_{N}\right)=\sigma^{2}
$$

may be written

$$
\tilde{\lambda}_{T}=\theta_{T} \cdot\left[\mu_{\nu 2} \frac{\sigma^{2}}{\|g\|_{2 v}} \cdot \frac{1}{N}\right]^{2 \nu /(4 \nu+1)}[1+o(1)] \text { as } N \rightarrow \infty,
$$

where

$$
\rho^{-4 \nu /(4 \nu+1)} C^{-2} \leqslant \theta_{T} \leqslant \rho^{4 \nu /(4 \nu+1)} c^{-2} .
$$

Under Axioms $\mathrm{A}$ and $\mathrm{C}$, we have instead

$$
\tilde{\lambda}_{T} \geqslant \theta_{T}\left[\mu_{\nu 2} \cdot \frac{\sigma^{2}}{\|g\|_{\nu}} \cdot \frac{1}{N}\right]^{2 \nu /(2 \nu+1)}[1+o(1)] \text { as } N \rightarrow \infty,
$$

where

$$
\rho^{-2 \nu /(2 \nu+1)} C^{-2} \leqslant \theta_{T} \leqslant \rho^{2 \nu /(2 \nu+1)} c^{-2} .
$$

We may deduce from (3.10) and (4.16) that under strong regularity of $g$,

$$
\rho^{-2}\left(4 \nu \mu_{\nu 2} / \kappa_{\nu}\right)^{2 \nu /(4 \nu+1)}[1+o(1)] \leqslant \lambda_{T} / \lambda_{0} \leqslant \rho^{2}\left(4 \nu \mu_{\nu} / \kappa_{\nu}\right)^{2 \nu /(4 \nu+1)}[1+o(1)] \text {. }
$$

Clearly $\lambda_{T}$ is weakly $S$-optimal. When $\rho=1$ we have

$$
\lambda_{T}=\left(4 \nu \mu_{\nu 2} / \kappa_{v}\right)^{2 \nu /(4 v+1)} \bar{\lambda}_{0}[1+o(1)]
$$

which again oversmooths relative to $\tilde{\lambda}_{0}$, but not so much as $\tilde{\lambda}_{D}$. Again, under weak regularity we have weak $S$-optimality if the rates in (3.10) and (4.18) are achieved.

The above two methods suffer from the drawback that a knowledge of $\sigma^{2}$ is necessary before they can be implemented. Even when $\sigma^{2}$ is known exactly, which is rare in practical situations, the methods are no better than weakly $S$-optimal. Another disadvantage is that they cannot be used to optimize $p$. One way of overcoming this difficulty is simply to minimize $\tilde{D}\left(\boldsymbol{\alpha} ; \mathbf{y}_{N}\right)$ with respect to $\alpha$, but this does not appear to have been tried. Such an approach would inevitably lead to

$$
\min _{\boldsymbol{\alpha}} \tilde{D}\left(\boldsymbol{\alpha} ; \mathbf{y}_{N}\right)<\boldsymbol{\sigma}^{2}
$$


so that the basic philosophy of the discrepancy principle would have to be abandoned. (See also the method of Klein [12].)

\section{Practical optimizing methods for $\alpha$ when $\sigma^{2}$ is unknown}

\subsection{Cross-validation}

Suppose we ignore the $j$ th data point $y_{j}$ and define the regularized solution $f_{N ; \alpha}^{[j]} \in T_{M}$ as the minimizer of

$$
\frac{1}{N} \sum_{\substack{n=0 \\ n \neq j}}^{N-1}\left[(K f)\left(x_{n}\right)-y_{n}\right]^{2}+\lambda\left\|f^{(p)}\right\|^{2}
$$

The $j$ th element of the predictive signal vector $\mathbf{g}_{N ; \alpha}^{[j]}=K_{N} \mathbf{f}_{N ; \alpha}^{[j]}$ predicts the missing datum $y_{j}$. We may thus choose an $\alpha$ which minimizes a weighted mean-square prediction error over all $j$ :

$$
V_{C V}(\alpha)=\frac{1}{N} \sum_{j=0}^{N-1} w_{j}(\alpha)\left[g_{N ; \alpha, j}^{[j]}-y_{j}\right]^{2}
$$

Wahba [20] has shown, in a general context, that if $A(\alpha)$ is the influence matrix of the regularized problem, then the choice of weights

$$
w_{j}(\alpha)=\left[\frac{1-A_{j j}(\alpha)}{\frac{1}{N} \operatorname{Trace}(I-A(\alpha))}\right]^{2}, \quad j=0, \ldots, N-1
$$

enables (5.1) to be expressed very simply in the form

$$
V_{C V}(\alpha)=\frac{\frac{1}{N}\left\|(I-A(\alpha)) \mathbf{y}_{N}\right\|^{2}}{\left[\frac{1}{N} \operatorname{Trace}(I-A(\alpha))\right]^{2}} .
$$

In our model problem the matrix $A(\alpha)$ is circulant and the weights in (5.1) all unity. From (2.22) and (2.24) we have

$$
V_{C V}(\alpha)=\frac{\frac{1}{N} \sum_{-M}^{M \prime \prime}\left(1-Z_{q ; \alpha}\right)^{2}\left|\hat{y}_{N, q}\right|^{2}}{\left[\frac{1}{N} \sum_{-M}^{M}\left(1-Z_{q ; \alpha}\right)\right]^{2}} .
$$

In principle it is an easy matter to minimize $V_{C V}(\lambda)$ for fixed $p$, and to repeat the process for a sequence of $p$-values until a minimizing pair $(p, \lambda)$ of $V_{C V}(\alpha)$ is found. In practice the method can work spectacularly well provided there is sufficient data, the problem is not too severely ill-posed, and the noise level is 
moderate. (See [8].) The minimization of (5.3) does not require an a priori knowledge of $\sigma^{2}$.

When $p$ is fixed the following result may be found in [20] (for the case of strong regularity) and in [4] (for weak regularity):

Theorem 5.1 Under Axioms A and B or A and C the minimizer $\tilde{\lambda}_{C V}$ of $E V_{C V}(\lambda)$ satisfies

$$
\tilde{\lambda}_{C V}=\tilde{\lambda}_{0}(1+o(1)) \quad \text { as } N \rightarrow \infty
$$

and

$$
\frac{E S\left(\tilde{\lambda}_{C V}\right)}{E S\left(\tilde{\lambda}_{0}\right)}=1+o(1) \text { as } N \rightarrow \infty
$$

This means that $\lambda_{C V}$ is $S$-optimal under both strong and weak regularity of $g$. Even stronger results than (5.4)-(5.5) are possible under sufficient regularity [7].

Our comments at the end of $\S 3$ on the existence of an optimal $p$ which minimizes $E S(\alpha)$ are also relevant for the minimization of $E V_{C V}(\alpha)$.

\subsection{Maximum likelihood}

This idea was first used to estimate regularization parameters for ill-posed problems by Anderssen and Bloomfield [1] in the case of numerical differentation. Extensions to numerical deconvolution were made by Anderssen and Prenter [2] and by Davies [6]. More recently Wahba [21] has suggested a generalization which can treat more general integral equations of the first kind.

For our model problem it is sufficient to assume that the data function and the errors are independent stationary stochastic processes with spectral density functions $P_{g}(\zeta)$ and $P_{\varepsilon}(\zeta)$, respectively. The regularization filter in $(2.19)$ can be formally equivalenced with a Wiener filter parametrized by $\alpha$ :

$$
Z_{q ; \boldsymbol{\alpha}}=\frac{P_{g}\left(\zeta_{q}\right)}{P_{g}\left(\zeta_{q}\right)+P_{e}\left(\zeta_{q}\right)}, \quad \zeta_{q}=\frac{\omega_{q}}{N}
$$

In terms of the unknown variance $\sigma^{2}$ we may write

$$
P_{\varepsilon}\left(\zeta_{q}\right)=\sigma^{2}, \quad-M \leqslant q \leqslant M,
$$

and

$$
P_{y}\left(\zeta_{q}\right)=P_{g}\left(\zeta_{q}\right)+P_{\varepsilon}\left(\zeta_{q}\right)=\sigma^{2}\left(1-Z_{q ; \alpha}\right)^{-1} \text {. }
$$

The unknown values of $\sigma^{2}$ and $\alpha$ can be estimated from the data by maximizing the likelihood function proposed by Whittle [22] for parameters in a stochastic model. The estimates are

$$
\boldsymbol{\sigma}^{2}=\hat{\boldsymbol{\sigma}}^{2}(\hat{\boldsymbol{\alpha}})=\frac{1}{N-1} \sum_{q=-M}^{M}\left(1-Z_{q ; \boldsymbol{\alpha}}\right)\left|\hat{y}_{N, q}\right|^{2}
$$


and $\boldsymbol{\alpha}=\hat{\boldsymbol{\alpha}}$ where $\hat{\boldsymbol{\alpha}}$ is the minimizer of

$$
V_{M L}(\alpha)=\sum_{q=-M}^{M}\left(1-Z_{q ; \alpha}\right)\left|\hat{y}_{N, q}\right|^{2} /\left[\prod_{\substack{q=-M \\ q \neq 0}}^{M}\left(1-Z_{q ; \alpha}\right)\right]^{1 /(N-1)} .
$$

When $p$ is fixed we announce the following (cf. Theorem 5.1 in [7]):

Theorem 5.2. Under Axioms A and B or A and $\mathrm{C}$, the minimizer of $E V_{M L}(\lambda)$ may be written

$$
\tilde{\lambda}_{M L}=\theta_{M L} \cdot\left[\kappa_{\nu} \frac{\sigma^{2}}{\|g\|_{\nu}} \frac{1}{N}\right]^{2 \nu /(2 \nu+1)}[1+o(1)] \text { as } N \rightarrow \infty,
$$

where

$$
C^{-2} \leqslant \theta_{M L} \leqslant c^{-2} \text {. }
$$

Furthermore

$$
E S\left(\tilde{\lambda}_{M L}\right) \leqslant \phi_{M L} \cdot\left[\kappa_{\nu} \cdot \frac{\sigma^{2}}{N}\right]^{2 \nu /(2 \nu+1)}\|g\|_{\nu}^{1 /(2 \nu+1)}[1+o(1)],
$$

where

$$
\begin{aligned}
& R^{-1}\left(1+R^{-2 v}\right) \leqslant \phi_{M L} \leqslant R\left(1+R^{2 v}\right), \\
& R=\rho^{2 /(2 v+1)} .
\end{aligned}
$$

We may deduce that, under strong regularity

$$
\tilde{\lambda}_{M L} / \tilde{\lambda}_{0}=O\left(N^{-4 \nu^{2} /(2 v+1)(4 v+1)}\right) \text { as } N \rightarrow \infty,
$$

and

$$
E S\left(\tilde{\lambda}_{M L}\right) / E S\left(\tilde{\lambda}_{0}\right)=O\left(N^{2 v /(2 v+1)(4 v+1)}\right) \text { as } N \rightarrow \infty,
$$

which means that $\lambda_{M L}$ undersmooths relative to $\lambda_{0}$ and is $S$-suboptimal. Under weak regularity, however,

$$
\tilde{\lambda}_{M L} / \tilde{\lambda}_{0} \leqslant \theta \cdot(1+o(1))
$$

where

$$
\left[\rho^{-(12 v+2)}(4 \nu)^{2 \nu}\right]^{1 /(2 v+1)} \leqslant \theta \leqslant\left[\rho^{(12 v+2)}(4 \nu)^{2 \nu}\right]^{1 /(2 v+1)} .
$$

This means that $\lambda_{M L}$ can oversmooth relative to $\lambda_{0}$. If the rates in (3.10) are achieved then $\lambda_{M L}$ is weakly $S$-optimal.

There is some numerical evidence to suggest that maximum likelihood can equal the performance of cross-validation when $N$ is small $(\sim 32)$ and the degree of ill-posedness of the problem is not great $[8,21]$. There is also some suggestion that $V_{M L}(\alpha)$ may, on occaison, be less sensitive to changes in $p$ than $V_{C V}(\alpha)$. 
Davies and Anderssen [7] have shown that $\lambda_{M L}$ is optimal (in the sense of (3.3)) with respect to the innovation variance of the data. This means that when the data constitute a stationary stochastic process, $\lambda_{M L}$ minimizes the approximation error in a least-squares regression on past data.

\section{Acknowledgements}

This work was conceived while the first author was a Visiting Fellow at the Centre for Mathematical Analysis, The Australian National University, and C.S.I.R.O., Division of Mathematics and Statistics. The support and hospitality of the host institutions are gratefully acknowledged.

\section{References}

[1] R. S. Anderssen and P. Bloomfield, "Numerical differentiation procedures for non-exact data", Numer. Math. 22 (1974), 157-182.

[2] R. S. Anderssen and P. M. Prenter, "A formal comparison of methods proposed for the numerical solution of first kind integral equations”, J. A ustral. Math. Soc. Ser. B 22 (1981), 488-500.

[3] M. L. Baart, "Computation experience with the spectral smoothing method for differentiating noisy data", J. Comput. Phys. 42 (1981), 141-151.

[4] P. Craven and G. Wahba, "Smoothing noisy data with spline functions", Numer. Math. 31 (1979), 377-403.

[5] J. Cullum, “The effective choice of smoothing norm in regularization", Math. Comp. 33 (1979), 149-170.

[6] A. R. Davies, "On the maximum likelihood regularization of Fredholm convolution equations of the first kind", in Treatment of Integral Equations by Numerical Methods (eds. C. T. H. Baker and G. F. Miller) (Academic Press, London, 1982), 95-105.

[7] A. R. Davies and R. S. Anderssen, "Improved estimates of statistical regularization parameters in Fourier differentiation and smoothing", Research Report CMA-R01-85. Centre for Mathematical Analysis, Australian National University, 1985.

[8] A. R. Davies, M. Iqbal, K. Maleknejad and T. C. Redshaw, "A comparison of statistical regularization and Fourier extrapolation methods for numerical deconvolution" in Numerical treatment of inverse problems in differential and integral equations (eds. P. Deuflhard and $\mathrm{E}$. Hairer) (Birkhauser, Berlin, 1983), 320-334.

[9] H. A. Gamber, "Choice of an optimal shape parameter when smoothing noisy data", Comm. Statist. A-Theory Methods 8 (1979), 1425-1435.

[10] C. W. Groetsch, The theory of Tikhonov regularization for Fredholm equations of the first kind (Pitman, London, 1984).

[11] F. R. de Hoog, "Review of Fredholm equations of the first kind", in The application and numerical solution of integral equations (eds. R. S. Anderssen, F. R. de Hoog and M. A. Lukas) (Sijthoff and Noordhoff, 1980), 119-134.

[12] G. Klein, "On spline functions and statistical regularization of ill-posed problems", J. Comput. Appl. Math. 5 (1979), 259-263.

[13] M. A. Lukas, Regularization of linear operator equations, Ph.D. thesis, Australian National University, 1981.

[14] M. A. Lukas, “Convergence rates for regularized solutions”, 1984. To be published. 
[15] V. A. Morozov, "The error principle in the solution of operational equations by the regularization method", USSR Comput. Math. and Math. Phys. 8 (1968), 63-87.

[16] A. N. Tikhonov and V. Y. Arsenin, Solutions of ill-posed problems (trans. from the Russian, Wiley, New York, 1977).

[17] V. F. Turchin, "Solution of the Fredholm equation of the first kind in a statistical ensemble of smooth functions", USSR Comput. Math. and Math. Phys. 7 (1967), 79-96.

[18] V. F. Turchin, "Selection of an ensemble of smooth functions for the solution of the inverse problem", USSR Comput. Math. and Math. Phys. 8 (1968), 328-339.

[19] G. Wahba, "Smoothing noisy data by spline functions", Numer. Math. 24 (1975).

[20] G. Wahba, "Practical approximate solutions to linear operator equations when the data are noisy", SIAM J. Numer. Anal. 14 (1977), 651-667.

[21] G. Wahba, "A comparison of GCV and GML for choosing the smoothing parameter in the generalized spline smoothing problem", Technical Report No. 712. Dept. of Statistics, University of Wisconsin-Madison, 1983.

[22] P. Whittle, "Some results in time series analysis", Skand. Actuarietidskr. 35 (1952), 48-06.

\section{Appendix: A note on proofs}

The techniques of proof of the theorems in this paper are natural extensions of the techniques in [7]. From (2.19) and Axiom $A$ it can be seen that the regularization filters satisfy

$$
Z_{q ; \alpha}=\left(1+\lambda c_{q}^{2} \omega_{q}^{2 \nu}\right)^{-1}
$$

and

$$
1-Z_{q ; \alpha}=\lambda c_{q}^{2} \omega_{q}^{2 \nu} Z_{q ; \alpha}
$$

In [7], asymptotic estimates are obtained for the moment functions

$$
\begin{gathered}
\Omega_{r s}(\lambda)=\sum_{-M}^{M} \omega_{q}^{2 r} /\left(1+\lambda \omega_{q}^{2 \nu}\right)^{s}, \\
G_{r s}(\lambda)=\sum_{-M}^{M} \omega_{q}^{2 r}\left|\hat{g}_{N, q}\right|^{2} /\left(1+\lambda \omega_{q}^{2 \nu}\right)^{s}
\end{gathered}
$$

for numbers $r \geqslant 0$ and $s>0$. These can be used to obtain asymptotic estimates for the moments

$$
\begin{gathered}
\sum_{-M}^{M}{ }^{\prime \prime} c_{q}^{2 l} \omega_{q}^{2 r} Z_{q ; \alpha}^{s}=\beta_{l}^{r s}(\lambda) \Omega_{r s}\left(\lambda \eta^{r s}(\lambda)\right), \\
\sum_{-M}^{M}{ }^{\prime \prime} c_{q}^{2 l} \omega_{q}^{2 r}\left|\hat{g}_{N, q}\right|^{2} Z_{q ; \alpha}^{s}=\gamma_{l}^{r s}(\lambda) G_{r s}\left(\lambda \zeta^{r s}(\lambda)\right),
\end{gathered}
$$

$l>0, r \geqslant 0, s>0$, where $\beta_{l}^{r s}(\lambda), \gamma_{l}^{r s}(\lambda), \eta^{r s}(\lambda)$ and $\zeta^{r s}(\lambda)$ are bounded continuous functions of $\lambda$ satisfying

$$
\begin{aligned}
& c^{2 l} \leqslant \beta_{l}^{r s}, \gamma_{l}^{r s} \leqslant C^{2 l}, \\
& c^{2} \leqslant \eta^{r s}, \zeta^{r s} \leqslant C^{2} .
\end{aligned}
$$


The expressions (A5)-(A6) arise from elementary applications of the mean-value theorem, using the form of $Z_{q, a}$ in (A1) and the constraints on $c_{q}$ in Axiom A. Finally, the bounds on the constants $\theta$ and $\phi$ in all theorems come from the representations (A5)-(A6) which are subject to (A7)-(A8).

We illustrate these techniques by sketching the proofs of Theorems 3.1 and 3.2. All other theorems are proved in similar fashion, with the exception of Theorem 5.1 for which the reader should consult [20] and [4].

Proof of Theorem 3.1. Differentiating (A1) with respect to $\lambda$ gives

$$
\frac{\partial}{\partial \lambda} Z_{q ; \lambda}=-c_{q}^{2} \omega_{q}^{2 \nu} Z_{q ; \lambda}^{2} \text {. }
$$

Thus, using (4.4), and setting $\partial E S(\lambda) / \partial \lambda=0$, we find that the minimizer of $E S(\lambda)$ satisfies the nonlinear equation

$$
\lambda \sum_{q}^{\prime \prime} c_{q}^{4} \omega_{q}^{4 \nu}\left|\hat{g}_{N, q}\right|^{2} Z_{q ; \lambda}^{3}=\frac{\sigma^{2}}{N} \sum_{q}^{\prime \prime} c_{q}^{2} \omega_{q}^{2 \nu} Z_{q ; \lambda}^{3},
$$

or, using (A3)-(A4),

$$
\lambda=\frac{\beta_{1}^{\nu^{3}}(\lambda)}{\gamma_{2}^{2 \nu, 3}(\lambda)} \cdot \frac{\sigma^{2}}{N} \cdot \frac{\Omega_{v 3}\left(\lambda \eta^{\nu 3}(\lambda)\right)}{G_{2 \nu, 3}\left(\lambda \xi^{2 v, 3}(\lambda)\right)} .
$$

Under Axiom B, Lemmas 3.1 and 3.2 in [7] imply that

$$
\Omega_{\nu 3}(\lambda)=\frac{\kappa_{\nu}}{4 \nu} \cdot \lambda^{-(2 \nu+1) /(2 \nu)}\left[1-O\left(\left[N \lambda^{1 /(2 \nu)}\right]^{-4 \nu+1}\right)\right] \text { as } N \rightarrow \infty,
$$

and

$$
G_{2 \nu, 3}(\lambda)=\|g\|_{2 \nu}[1+o(1)] \text { as } N \rightarrow \infty,
$$

where $o(1) \rightarrow 0$. Equations (A11)-(A12) are valid provided $N \lambda^{1 /(2 v)} \rightarrow \infty$ as $N \rightarrow \infty$. Under this condition, rearrangement of (A10) yields

$$
\lambda^{(4 \nu+1) /(2 \nu)}=\beta_{1}^{\nu 3}\left(\gamma_{2}^{2 \nu, 3}\right)^{-1}\left(\eta^{\nu 3}\right)^{-(2 \nu+1) /(2 \nu)} \cdot \frac{\kappa_{\nu}}{4 \nu} \cdot \frac{\sigma^{2}}{\|g\|_{2 \nu}} \cdot \frac{1}{N}[1+o(1)]
$$

or alternatively, (3.10), with

$$
\theta_{0}=\left[\beta_{1}^{\nu 3} / \gamma_{2}^{2 \nu, 3}\right]^{(2 v) /(4 \nu+1)}\left(\eta^{\nu 3}\right)^{-(2 \nu+1) /(4 v+1)} .
$$

The bounds (3.11) follow from (A7)-(A8). It is a simple matter to confirm that $N \bar{\lambda}_{0}^{1 /(2 \nu)} \rightarrow \infty$ as $N \rightarrow \infty$.

Equation (3.13) and inequality (3.14) arise similarly by direct substitution of (3.10) and (A13) into (4.4).

Proof of Theorem 3.2. Here we replace (4.4) by the inequality

$$
E S(\lambda) \leqslant \frac{\sigma^{2}}{N} \sum_{q}^{\prime \prime} Z_{q ; \lambda}^{2}+\sum_{q}^{\prime \prime}\left(1-Z_{q ; \lambda}\right)\left|\hat{g}_{N, q}\right|^{2},
$$


since $0 \leqslant 1-Z_{q ; \lambda} \leqslant 1$. By differentiation, the right hand side of (A14) is minimized when

$$
\gamma_{1}^{\nu 2} G_{\nu 2}\left(\lambda \zeta^{\nu 2}\right)=\frac{2 \sigma^{2}}{N} \cdot \beta_{1}^{\nu 3} \Omega_{\nu 3}\left(\lambda \eta^{\nu 3}\right)
$$

Using Lemmas 3.1 and 3.2 in [7] we may deduce as before that the solution of (A15) satisfies

$$
\lambda=\theta\left[\frac{\kappa_{\nu}}{2 \nu} \cdot \frac{\sigma^{2}}{\|g\|_{\nu}} \cdot \frac{1}{N}\right]^{2 \nu /(2 v+1)}[1+o(1)]
$$

where

$$
\theta=\eta^{\nu}\left[\beta_{1}^{\nu 3}\left(\gamma_{1}^{\nu 2}\right)^{-1}\right]^{\nu /(2 \nu+1)}
$$

from which (3.17)-(3.18) follow. Finally, inequality (3.15) may be deduced from (A10) by observing that $\lambda G_{2 \nu, 3}(\lambda) \leqslant G_{\nu 2}(\lambda)$. 